\title{
A novel, high-performance random array platform for quantitative gene expression profiling
}

\author{
Kenneth Kuhn, ${ }^{1}$ Shawn C. Baker, ${ }^{1}$ Eugene Chudin, Minh-Ha Lieu, Steffen Oeser, \\ Holly Bennett, Philippe Rigault, David Barker, Timothy K. McDaniel, ${ }^{2}$ and \\ Mark S. Chee
}

Illumina, Inc., San Diego, California 92121, USA

\begin{abstract}
We have developed a new microarray technology for quantitative gene-expression profiling on the basis of randomly assembled arrays of beads. Each bead carries a gene-specific probe sequence. There are multiple copies of each sequence-specific bead in an array, which contributes to measurement precision and reliability. We optimized the system for specific and sensitive analysis of mammalian RNA, and using RNA controls of defined concentration, obtained the following estimates of system performance: specificity of 1:250,000 in mammalian poly( $\left(\mathrm{A}^{+}\right) \mathrm{mRNA}$; limit of detection $0.13 \mathrm{pM}$; dynamic range 3.2 logs; and sufficient precision to detect 1.3 -fold differences with $95 \%$ confidence within the dynamic range. Measurements of expression differences between human brain and liver were validated by concordance with quantitative real-time PCR $\left(R^{2}=0.98\right.$ for log-transformed ratios, and slope of the best-fit line $=1.04$, for 20 genes). Quantitative performance was further verified using a mouse B- and T-cell model system. We found published reports of B- or T-cell-specific expression for 42 of 59 genes that showed the greatest differential expression between B- and T-cells in our system. All of the literature observations were concordant with our results. Our experiments were carried out on a 96-array matrix system that requires only $100 \mathrm{ng}$ of input RNA and uses standard microtiter plates to process samples in parallel. Our technology has advantages for analyzing multiple samples, is scalable to all known genes in a genome, and is flexible, allowing the use of standard or custom probes in an array.
\end{abstract}

Microarray technology has allowed the abundance of thousands of different mRNAs to be measured simultaneously and efficiently from a single biological sample (Schena et al. 1995; Lockhart et al. 1996; Lockhart and Winzeler 2000). As a result, the analysis of individual genes has given way to the analysis of large sets of genes and the discovery of patterns and relationships in their expression. This has spawned a myriad of exciting new applications that are helping to shape the emerging field of systems biology (Marton et al. 1998; Golub et al. 1999; Hughes et al. 2000; Ideker et al. 2001; van't Veer et al. 2002; Yvert et al. 2003). The microarrays that have spurred these advances can be manufactured by a variety of techniques, including spotting (Schena et al. 1995), photolithographic synthesis (Fodor et al. 1991), and inkjet synthesis (Blanchard 1998). In each case, individual probes are placed or synthesized at predefined locations on the substrate. However, conventional arrays can suffer from one or more limitations, including poor data quality, as a result of high intraand interarray variability, often associated with spotted arrays.

We describe here a powerful and intrinsically robust alternative that substantially overcomes these limitations. Our geneexpression profiling system is based on randomly assembled arrays of beads in wells (Michael et al. 1998). Following random assembly, the location and identity of each bead, bearing an oligonucleotide probe, is determined via a sequential decoding process (Gunderson et al. 2004). An advantage of this approach is that dense packing can be achieved using simple and efficient

\footnotetext{
'These authors contributed equally to this work.

${ }^{2}$ Corresponding author.

E-mail tmcdaniel@illumina.com; fax (858) 202-4680.

Article and publication are at http://www.genome.org/cgi/doi/10.1101/ gr.2739104.
}

bulk processes. Furthermore, the technology is intrinsically scalable; the arrays described in this study use beads with diameters of three microns, producing a packing density $\sim 400$ times that of a conventional spotted microarray. Elsewhere, packing densities $\sim 40,000$ times that of a conventional array have been achieved through the assembly of 300-nm beads (Michael et al. 1998).

The BeadArray technology has previously been shown to be a robust readout platform for single nucleotide polymorphism (SNP) genotyping, where it has demonstrated very high accuracy, call rate, and reproducibility at high multiplexing levels (Fan et al. 2003). It is being used to generate over half the genotyping data for the International HapMap Project (www.hapmap.org), which will derive a detailed map of common genetic variation across the human genome (The International HapMap Consortium 2003). In addition, the BeadArray platform has been effective for gene-expression profiling using PCR-based assays in combination with universal arrays (Yeakley et al. 2002; Fan et al. 2004). Both of these applications made use of universal arrays containing up to 1536 usable capture sequences.

Despite these successes, the use of the BeadArray technology for quantitative gene-expression profiling from complex samples by hybridization to gene-specific probes has not previously been demonstrated. Although some preliminary data suggested that the platform is capable of high sensitivity (Epstein et al. 2002), a number of significant challenges had to be overcome in order to create a robust, quantitative, high-performance system suitable for use with biological samples such as mammalian poly $\left(\mathrm{A}^{+}\right)$ mRNA. We have now developed such a system. We show that it is capable of accurately and robustly reporting mRNA abundance for hundreds of genes. Our methods can be applied to many thousands of samples, a scale of experimentation that has been 
impractical with other technologies, and can be extended to develop arrays designed to analyze all known genes in a genome. The technology is also compatible with our SNP genotyping system, enabling genotyping and gene-expression profiling on the same platform.

\section{Results}

\section{Design of a gene-expression probe array based on random} assembly of beads in wells

The arrays used in the experiments reported here are described in Figure 1. Typically, each array has up to 1536 different bead types, each represented on average by $\sim 30$ copies in any array. Each bead type has $\sim 700,000$ copies of a particular oligonucleotide probe covalently attached to it (Fig. 1). Because the population of beads in an array is a random sampling of a starting bead pool containing 1536 bead types, the representation of the bead types in the array is effectively Poisson. That is, there is a variable number of each of the 1536 bead types both within and between arrays (Gunderson et al. 2004). Thus, two important issues must be addressed to ensure that the random arrays can be used for quantitative measurements of mRNA abundance.

Firstly, because each array is unique, how can we compare results from array to array? By virtue of the $\sim 30$-fold oversampling (50,000 beads/1536 bead types), we can ensure that decoded arrays have greater than or equal to five beads of each type in the array, so that all sequences are represented (Gunderson et al. 2004). Furthermore, the randomness and redundancy provide us with considerable advantages; randomness minimizes the effects of spatially localized artifacts, and redundancy increases measurement precision and robustness. These factors combine to increase measurement accuracy.

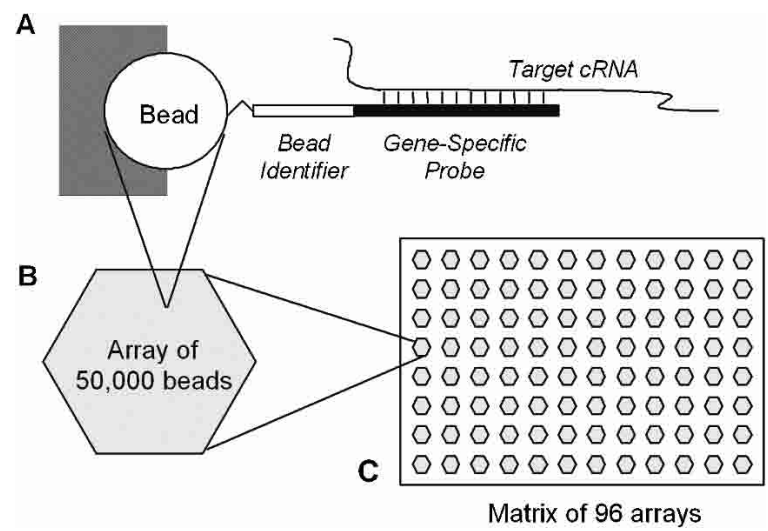

Figure 1. Design of a randomly assembled gene-specific probe array. $(A)$ Representation of an individual bead lodged in a well. Attached to the bead by its $5^{\prime}$ end is a chimeric oligonucleotide $\sim 75$ nucleotides in length, comprising an $\sim 25$-nucleotide identifier sequence and a 50-nucleotide gene-specific probe. The bead identifier sequence is decoded using an algorithm described previously (Gunderson et al. 2004). We tested genespecific probes of 25 and $50 \mathrm{bp}$ in length and found that the 50 mers showed superior performance, consistent with prior findings (Hughes et al. 2001). The drawing is not to scale; the relative size of the oligonucleotide has been vastly exaggerated to show its features. $(B)$ There are $\sim 50,000$ beads in an $\sim 1.4-\mathrm{mm}$ diameter optical fiber bundle, each bead lodged in a well at the end of an individual fiber in the bundle. ( $C$ ) The bundles are arranged in a 96-array matrix matching the format of a standard microtiter plate.
Secondly, because each probe has associated with it an identifier sequence (Fig. 1), how do we ensure that this sequence doesn't interfere with the analysis of the target mRNA? This is done in two ways. The identifier sequences are computationally screened to avoid similarity to the human and mouse genomes. The probability of cross-hybridization to other genomes is also low, and for the analysis of any particular genome, it is simple to omit a small number of identifier sequences if needed. Also, the identifier sequences are only half the length of the gene-specific probes and have correspondingly lower Tm's $\left(52.0 \pm 2.3^{\circ} \mathrm{C}\right.$ vs. $\left.70.7 \pm 1.7^{\circ} \mathrm{C}\right)$. By hybridizing labeled total mammalian poly $\left(\mathrm{A}^{+}\right)$ mRNA samples to arrays containing the identifier sequences but lacking the gene-specific probe sequences, we estimated that the identifier sequences contribute an average of up to five counts over background, with only a few sequences giving higher signals (data not shown). This is a small amount of signal relative to the gene-specific probes and is not expected to have any significant effect on the analysis.

\section{Array formats designed for a variety of gene-expression applications}

The experiments described in this study all make use of the Sentrix array matrix format shown in Figure 1. However, the basic concept of placing beads in wells to form a randomly ordered array can be used to create a variety of array formats suitable for a range of applications. In addition to the format shown in Figure 1 , which is read using a custom high-resolution reader (Barker et al. 2003), we have developed silicon substrates that have the dimensions of a $2.5 \times 7.5-\mathrm{cm}$ microscope slide and can be read on a $5-\mu \mathrm{m}$ resolution Axon GenePix scanner by virtue of larger well spacing (T. Dickinson, G. Smith, H. Bennett, and R. Barrett, unpubl.). Yet other silicon substrates have been used to develop two designs of whole-genome array, with probes for $\sim 24,000$ and $\sim 48,000$ gene sequences (G. Wang, G. Smith, S. Barnard, and D. Che, unpubl.; further information is available on www.illumina. com). These higher density arrays can be read on a BeadArray scanner. All of these formats make use of 3- $\mu \mathrm{m}$ silica beads; the same bead pools can be loaded into the different substrates, and give similar quantitative performance. Therefore, substantially similar results to those obtained below can be obtained using a variety of bead-based array formats suitable for a range of experimental designs and detection systems.

\section{Dose-response study using spiked mRNAs of known concentration}

We designed a dose-response study to estimate the limit of detection, dynamic range, and precision of the 96-array matrix gene-specific probe system for the analysis of a mammalian mRNA sample. We prepared a series of samples that consisted of labeled human liver cell line RNA spiked with known quantities of individually labeled mRNAs synthesized in vitro. This approach has been described previously for microarray performance characterization (Lockhart et al. 1996). We used as spikes nine mRNAs, produced by in vitro transcription (IVT) of cloned bacterial and viral genes whose sequences are absent from the human genome. Twelve samples, representing 12 concentrations, were each replicated eight times to give a total of 96 samples. Each sample contained all nine spikes at a given concentration ranging from zero to 200 pM. (Fig. 2).

Each sample was hybridized to eight different arrays in a 96-array matrix. This provided eight technical replicates, suffi-

\section{Genome Research}

www.genome.org 


\section{Random arrays for gene expression profiling}

\begin{tabular}{|c|c|c|c|c|c|c|c|c|c|c|c|c|}
\hline & 1 & 2 & 3 & 4 & 5 & 6 & 7 & 8 & 9 & 10 & 11 & 12 \\
\hline 1 & 200 & 100 & 30 & 15 & 10 & 3 & 1.5 & 1 & 0.3 & 0.15 & 0.1 & 0 \\
\hline 2 & 0 & 200 & 100 & 30 & 15 & 10 & 3 & 1.5 & 1 & 0.3 & 0.15 & 0.1 \\
\hline 3 & 0.1 & 0 & 200 & 100 & 30 & 15 & 10 & 3 & 1.5 & 1 & 0.3 & 0.15 \\
\hline 4 & 0.15 & 0.1 & 0 & 200 & 100 & 30 & 15 & 10 & 3 & 1.5 & 1 & 0.3 \\
\hline 5 & 0.3 & 0.15 & 0.1 & 0 & 200 & 100 & 30 & 15 & 10 & 3 & 1.5 & 1 \\
\hline 6 & 1 & 0.3 & 0.15 & 0.1 & 0 & 2000 & 100 & 30 & 15 & 10 & 3 & 1.5 \\
\hline 7 & 1.5 & 1 & 0.3 & 0.15 & 0.1 & 0 & 200 & 100 & 30 & 15 & 10 & 3 \\
\hline 8 & 3 & 1.5 & 1 & 0.3 & 0.15 & 0.1 & 0 & 200 & 100 & 30 & 15 & 10 \\
\hline
\end{tabular}

Figure 2. Arrangement of spiked samples for hybridization. Each sample was produced by adding labeled spike controls to labeled complex RNA derived from human HepG2 poly $\left(\mathrm{A}^{+}\right) \mathrm{RNA}$. The spike controls were added at the pM concentrations indicated in the figure. All nine spiked mRNAs were present at the same concentration within a given sample (e.g., 200 pM in sample a1). Samples were arranged in a staggered fashion to avoid the possibility of row/column positional bias. Hybridization was performed using $1 \mu \mathrm{g}$ of each sample at a final concentration of $25 \mathrm{ng} / \mu \mathrm{L}$.

cient data to allow a statistical analysis of noise in the quantitative readout step. The dose response curves and the resolvable fold change across the tested concentration range, generated for each of the nine genes, are shown in Figure 3.
Reproducibility of quantitative measurements and dependence on sample input

The dose-response results were reproducible across different manufacturing lots of array matrices and hybridization days. We obtained similar results from 15 independent trials of the experiment, hybridized on five separate days using a total of 720 arrays manufactured on seven different dates (Fig. 4). The quantitative performance of the system based on this significant amount of replication is summarized in Table 1.

In addition to the probes used to measure the dose responses, the arrays used in the experiments summarized in Figure 4 contained probes for 587 human genes. We analyzed the data generated by these experiments to assess array-
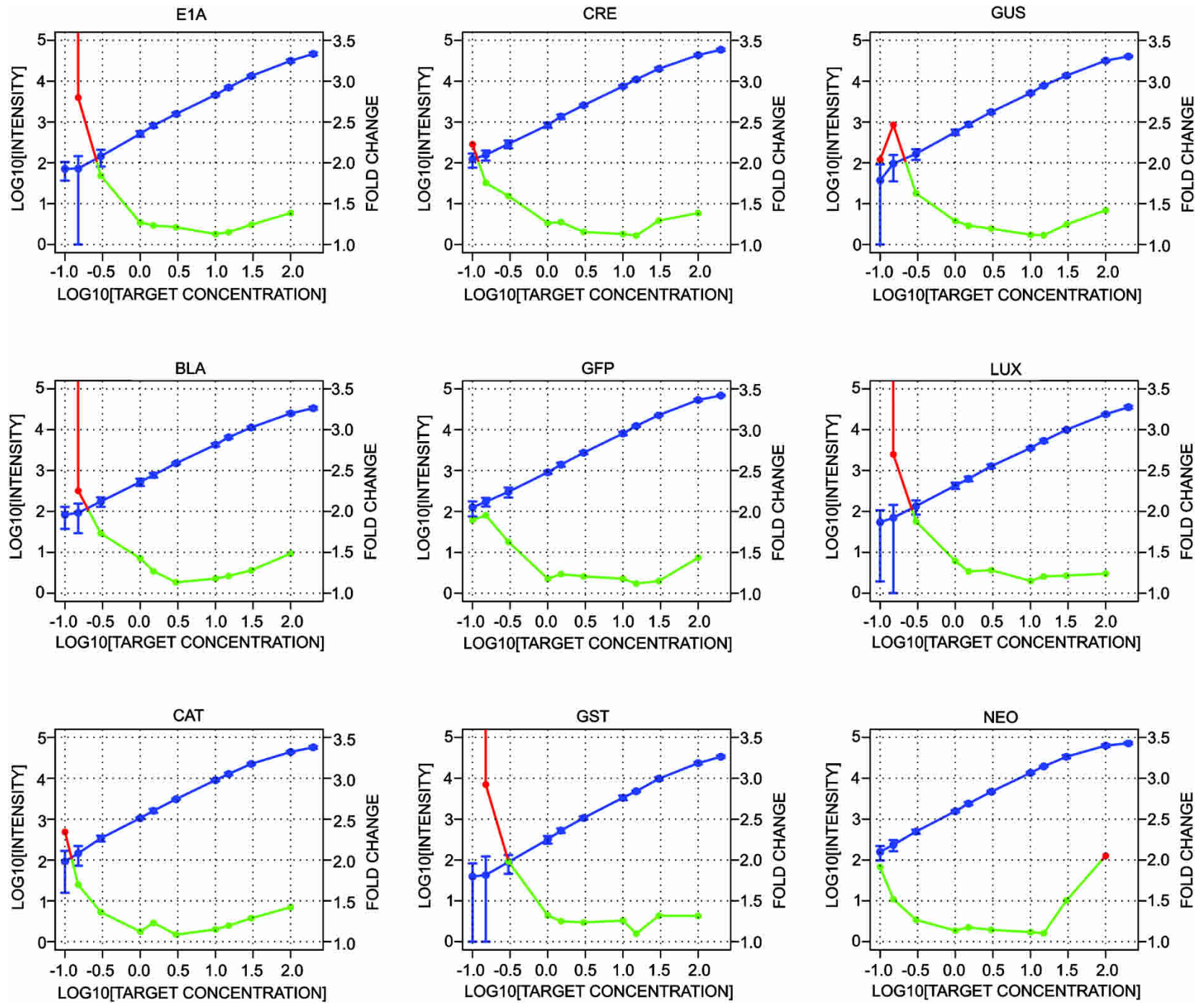

Figure 3. Dose-response curves. Data points represent the mean of eight arrays. Signal intensities are plotted in blue vs. target concentration. Error bars represent the two-sided symmetric $90 \%$ confidence intervals for a single reading, calculated on the basis of the spread of eight separate readings. All points contain error bars, but some are too small to be resolved at the plotted scale. The resolvable fold change is plotted in red and green vs. target concentration. Each data point estimates the ability to distinguish concentration fold change for a single reading. Concentration levels are defined as resolved when estimated one-sided 95\% confidence intervals do not overlap. Values below twofold are colored green, whereas those greater than twofold are colored red. 


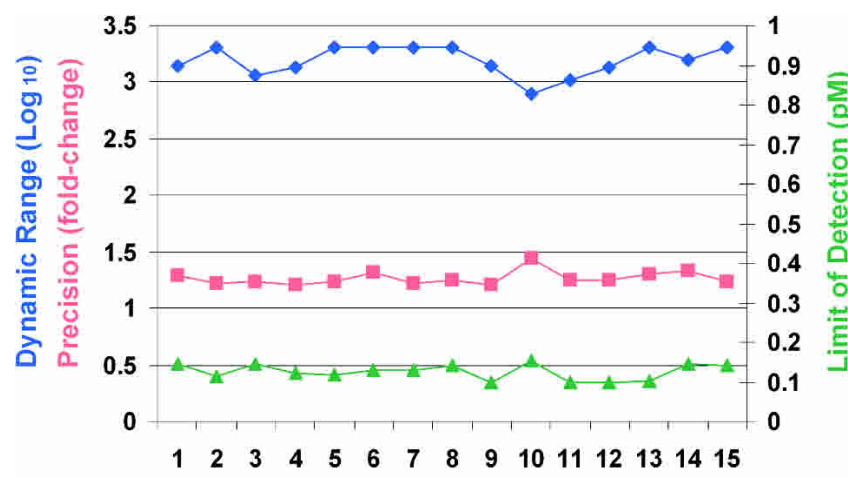

Array Matrix Number

Figure 4. Dynamic range, detectable fold change, and limit of detection for 15 array matrices. The array matrices, manufactured on five separate days, were used to perform dose-response experiments identical to that described above, except in these experiments, we used four replicates per concentration instead of eight. Dynamic range corresponds to the concentration range over which twofold concentration changes can be distinguished with $95 \%$ confidence (represented by the green portions of the lines in Fig. 3); the values plotted in the graph (blue diamonds, left axis) are determined by dividing the upper concentration limit of this range by the lower limit for the given experiment. Precision (orange squares, left axis) corresponds to the distinguishable fold change across the determined dynamic range. Limit of detection (green triangles, right axis) corresponds to 0.99 detection p-value generated using normal model of intensities of 20 negative control probes that have no corresponding target in the sample. All performance values given represent the median value for the nine spike targets used in the experiment.

to-array hybridization signal variation and how it is influenced by gene intensity. We selected the 380 genes that were reproducibly expressed at detectable levels and plotted their coefficient of variation (standard deviation divided by intensity, abbreviated as $\mathrm{CV}$ ) as a function of hybridization signal. As shown in Figure 5, and consistent with expectations, the $\mathrm{CV}$ increases inversely as gene signals approach the limit of detection. The median CV for

Table 1. Performance metrics

\begin{tabular}{lcc}
\hline Metric $^{\mathbf{a}}$ & Value & Confidence $^{-}$ \\
\hline Input Requirement (Total RNA) $^{\text {Limit of Detection }}{ }^{\mathrm{b}}$ & $100 \mathrm{ng}$ & $\mathrm{n} / \mathrm{a}$ \\
Specificity $^{\mathrm{c}}$ & $\sim 0.13 \mathrm{pM}$ & $99 \%$ \\
Precision $^{\mathrm{d}}$ & $\sim 1: 250,000$ & $99 \%$ \\
Dynamic Range $^{\mathrm{e}}$ & $\sim 1.3-$ fold & $95 \%$ \\
Array-to-Array \%CV $^{\mathrm{f}}$ & $\sim 3.2 \mathrm{Logs}$ & $\mathrm{n} / \mathrm{a}$ \\
& $<10 \%$ & $\mathrm{n} / \mathrm{a}$ \\
\hline
\end{tabular}

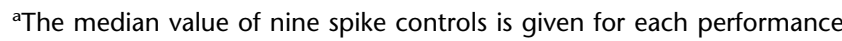
metric. All metrics listed derive from measurements made using two probe sequences per gene.

bimit of detection is determined by a negative control detection model (see Methods).

'Specificity is determined by dividing the number of molecules detected at the limit of detection by the number of molecules present in $1 \mu \mathrm{g}$ of sample background. The average mammalian transcript length is estimated at $2000 \mathrm{nt}$.

dPrecision is the smallest change in concentration that can be detected with $95 \%$ confidence. Value given is the median across the dynamic range.

eDynamic range is defined by the ability to detect twofold changes in target concentration across the specified concentration range (see Methods).

${ }^{\mathrm{f}}$ Array-to-array $\% \mathrm{CV}$ is determined using a common population of all detectable bead-types across the 96 arrays of an array matrix.

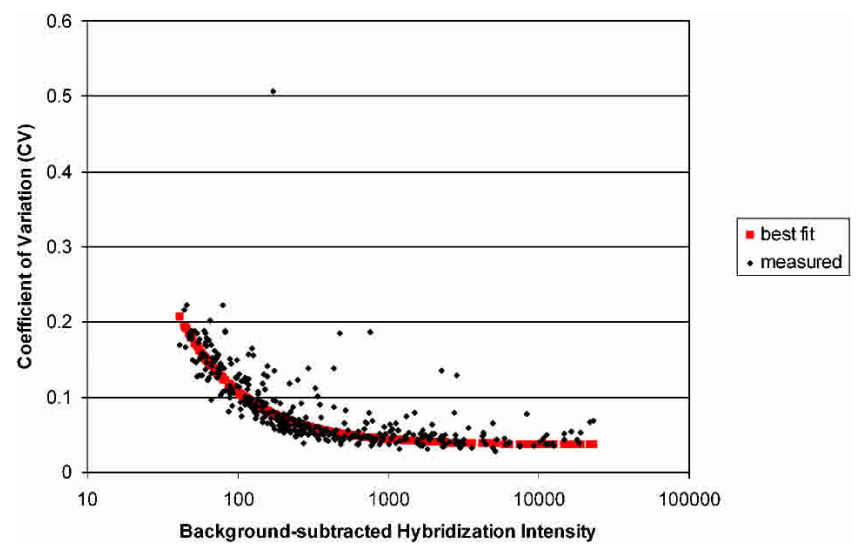

Figure 5. Array signal variation as a function of gene hybridization intensity. Each blue dot represents a gene and the red line represents a smoothed function for the data on the basis of a robust best-fit function for standard deviation vs. intensity. All values are based on backgroundsubtracted raw data from 48 replicate hybridizations. The data shown are from one experiment of the 15 described in the legend to Figure 4. This experiment was chosen to represent the others on the basis of its measurement precision, which is the median of measurement precisions for all 15 experiments.

background-subtracted, un-normalized intensity across 48 arrays in a representative experiment was $6.5 \%$.

Additional performance measurements of a microarray platform include (1) reproducibility across multiple sample labeling reactions, and (2) sensitivity to sample input variation. To test these aspects of our system's reproducibility, we performed 20 sample labeling reactions, four each, using 10, 20, 50, 150, or 500 ng of total RNA derived from mouse spleen. For the 10- and 20-ng inputs, only three of the four replicates produced adequate material for array hybridization. One microgram of biotinylated cRNA from each successful reaction was hybridized to a separate array in an array matrix. Each array in the matrix contained probes to 540 mouse genes. Each cRNA sample was present at a final concentration of $25 \mathrm{ng} / \mu \mathrm{L}$.

To obtain a quantitative estimate of reproducibility, linear correlations were calculated for all pairwise combinations of the replicates at each input concentration. The means and ranges of these correlations are plotted in Figure 6A. All correlations $\left(\mathrm{R}^{2}\right)$ exceeded 0.99. As further evidence of robustness, the scatter plot in Figure 6B shows the correlation for signals between sample labeling replicates using 50 and $500 \mathrm{ng}$ of starting material; the high correlation $\left(\mathrm{R}^{2}>0.99\right)$ demonstrates the reproducibility of the assay even with input material concentrations differing by 10 -fold.

\section{Concordance with real-time quantitative PCR}

The experiments described above measured the quantitative performance of the system and demonstrated that we could obtain quantitative data in a reproducible way. We next wanted to perform measurements on a true biological sample and to evaluate these results by comparison with a different technology. Concordance with measurements obtained using a different technology is a strong indicator that measurements are correct. Therefore, we performed an experiment that compared differential expression patterns obtained on the randomly assembled arrays with those obtained from TaqMan quantitative real-time PCR (qPCR).

The genes selected for this analysis came from a comparison

\section{Genome Research}

www.genome.org 
A

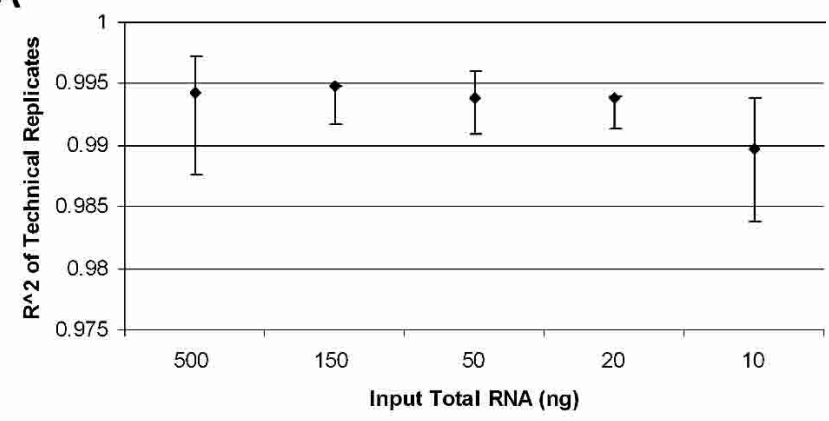

B

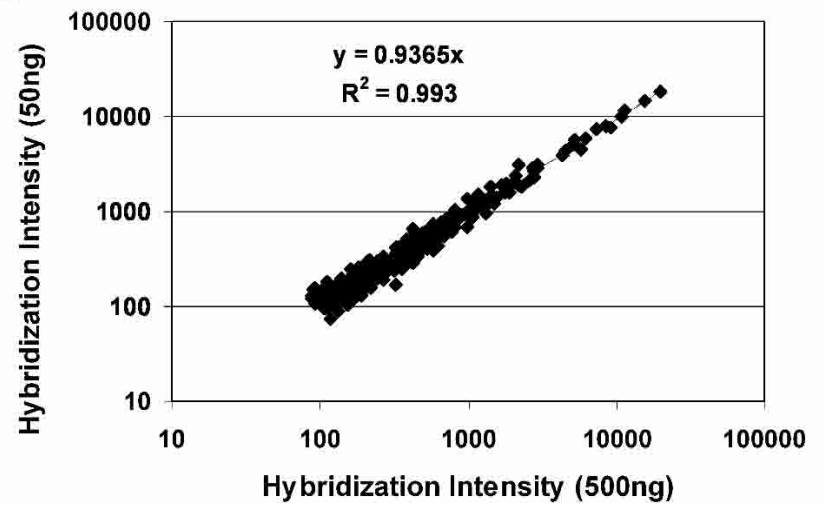

Figure 6. Sample labeling reproducibility. (A) Twenty sample labeling reactions were processed using our standard conditions with 10,20,50, 150 , or $500 \mathrm{ng}$ of total mouse spleen RNA as input material (four replicates each). For each input amount, correlation values $\left(\mathrm{R}^{2}\right)$ of gene signals were determined for all pairwise comparisons of all successful replicates. The median $\mathrm{R}^{2}$ values, with ranges, are plotted. (B) Representative scatter plot of the intensity values for all genes measured in one of the 50 -ng samples vs. those in the 500-ng sample. Whereas initial sample input varied for each labeling reaction, final array hybridization was performed using $1 \mu \mathrm{g}$ of each labeled sample at a final concentration of 25 $\mathrm{ng} / \mu \mathrm{L}$.

of human liver with human brain. Labeled cRNA from both tissues was hybridized to separate arrays containing probes for 633 human genes. From the hybridization results, we selected a panel of 21 genes for the comparison, using the following criteria: (1) the genes showed a range of liver/brain expression ratios ranging from 0.005 to 175 ; and (2) every gene was expressed significantly over background, even in the tissue showing the lower amount of expression. This second criterion was necessary to avoid inaccurate expression ratios resulting from the influence of system noise.

For each of these 21 genes, we performed qPCR assays on aliquots of the same starting material. Twenty of the 21 primer pairs gave products and the log-transformed expression ratios obtained for each of the 20 genes were plotted against the corresponding values obtained on the randomly assembled arrays (Fig. 7). The measurements determined by the two systems showed good correlation $\left(\mathrm{R}^{2}=0.98\right.$ for log-transformed ratios). Furthermore, the slope of the best-fit line was 1.04, indicating that the ratios obtained by the two methods are similar in magnitude. For highly expressed genes, the array produced somewhat compressed fold-change ratios compared with those produced by qPCR. For the five genes whose array intensities exceeded 10,000 counts in either tissue, the array-measured ratio was $0.77 \pm 0.24$ versus $1.04 \pm 0.35$ for all genes. This compression is likely due to probe saturation of highly expressed targets, a predicted feature, as the array platform has a dynamic range of $\sim 3$ logs compared with $\sim 5$ logs for qPCR. (Heid et al. 1996) This overall high level of concordance with qPCR validated the performance of the randomly assembled array system.

\section{Validation of results in a model biological system}

Finally, we assessed the ability of the random arrays to generate data consistent with results previously published for a wellcharacterized biological system. The model system we selected was mouse B and T cells, both of which contain large numbers of cell-type specific transcripts documented in the biological literature. Our experimental design was to make a series of seven samples containing different ratios of R1.1 (T cell lymphoma) and A20 (B cell lymphoma) mRNA mixed together. This series ranged from $100 \% \mathrm{~B} / 0 \% \mathrm{~T}$ to $0 \% \mathrm{~B} / 100 \% \mathrm{~T}$. Each of the seven samples was independently labeled six times, and the resulting 42 cRNA samples were hybridized to separate arrays of an array matrix, each containing probes to 540 different mouse genes. After hybridization and analysis, we identified 59 genes that were determined as detected in the $100 \%$ B cell sample, but not in the $100 \%$ T cell sample or vice versa (Fig. 8). Upon generating this list of 59 genes, we performed literature searches to establish whether there was prior evidence of T- or B-specific expression. Forty three of the 59 genes had prior literature support for their tissue specificity. We found no genes miscategorized by our array. Table 2 shows a list of all tissue-specific genes identified in our analysis.

\section{Discussion}

We developed a powerful and robust new microarray technology for gene-expression profiling on the basis of randomly assembled arrays of beads in wells. The high information density of these arrays ( 50,000 beads/ 1.4-mm diameter array) reduces sample consumption and makes them well suited for integration into sophisticated systems such as the array matrix device described herein. Each probe is replicated a minimum of five times and on average $\sim 30$ times on every array. This built-in redundancy increases measurement precision and makes for an intrinsically robust measurement platform. We optimized the system for hybridization specificity and sensitivity, integrated the various components into a scalable system for gene-expression quantitation, and showed that accurate and reproducible data are generated from complex biological samples.

The 96-array matrix format and associated protocols make it straightforward to analyze many samples with relatively little labor and high reproducibility. We consider this a significant advance because sources of noise and error, such as intra- and interarray variability, process variability, and biological sample variability, can confound microarray experiments (Brody et al. 2002). An effective way of identifying, characterizing, and minimizing variation is to apply well-known statistical tools. Unfortunately, the ease-of-handling, and in many cases, the reproducibility of current microarray technologies makes it difficult to replicate experiments adequately. This has severely limited the ability to generate and analyze large data sets. As a consequence, the use of microarrays in applications requiring the analysis of large numbers of samples, such as epidemiological, toxicological, 
A

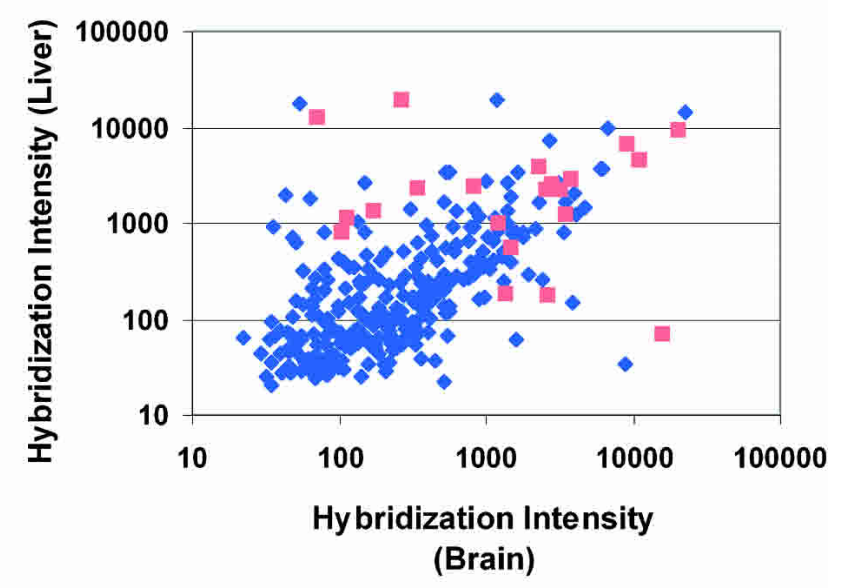

B

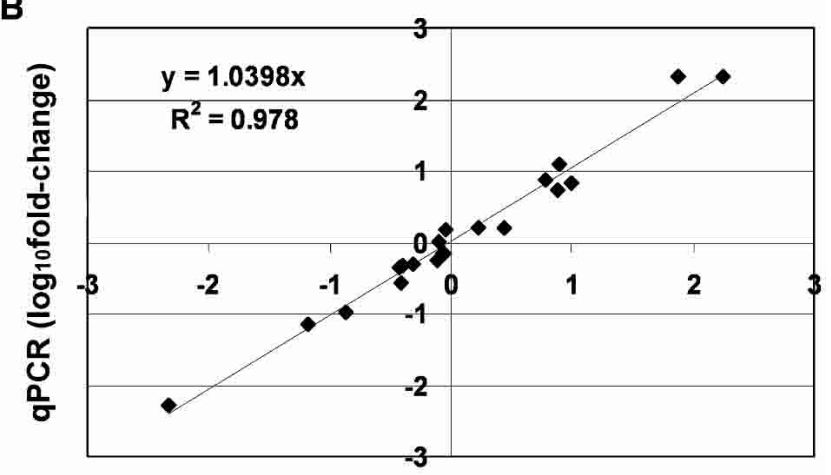

Array Matrix ( $\log _{10}$ fold-change)

Figure 7. Correlation of array matrix data to quantitative real-time PCR. Labeled RNA samples were made from human and brain total RNA. These were hybridized to separate array matrices containing 633 human genes. Six technical replicates were included for each sample. Twentyone genes from this list were selected for analysis by TaqMan quantitative real-time PCR. A scatter plot of hybridization intensities of the liver and brain samples on the array matrix is shown in $A$. Genes selected for further analysis are shaded orange. A scatter plot of log-transformed hybridization signal ratios as determined by the two methods is shown in $B$.

and pharmacological screening, has been limited mostly to proof-of-concept studies. Meaningful application of highthroughput microarray technology to large sample sets is now more practical as a result of the system described here.

Samples can be processed in standard micro-plate formats, either manually or robotically. The entire system is designed for compatibility with automation and LIMS tracking, and hence, is suitable for use in applications that require a highly reproducible process with accurate sample tracking throughout. The technology is flexible. It can be used to analyze the expression of hundreds of genes, as described in this study, as well as wholegenome sets of many thousands of genes, which will be described elsewhere. The ability to assemble large numbers of arrays from a single bead pool on the basis of a common chemistry helps to minimize interarray variability. Flexibility in array design is provided by the ability to supplement standard bead pools with sequences of the user's choosing or to make custom bead pools. ${ }^{3}$

We also developed software for array imaging and geneexpression data analysis (E. Chudin and I. Mikouliteh, unpubl.).
Because of the robustness of the system, the user has to pay less attention to the data extraction process than typical with spotted arrays, and can instead focus on analysis of results. AnEx, a geneexpression data analysis program that organizes sample data and incorporates statistical and visualization tools, is commercially available as part of the gene-expression analysis system. AnEx is MIAME-compliant (www.mged.org) and generates a flat-file format that is accepted by many third-party analysis software applications.

Finally, an advantage of the system we have developed is that it uses the same technology platform as our SNP genotyping system (Fan et al. 2003) and our PCR-based gene-expression assay system (Fan et al. 2004). As a result, SNP genotyping and geneexpression profiling can now be carried out on a single microarray platform, scalable from the analysis of hundreds of genes to all known genes in a genome.

\section{Methods}

\section{Samples}

Human brain and liver total RNA were purchased from Ambion (Cat. \#7962, Brain; 7960, Liver). Human HepG2 Poly(A $\left.{ }^{+}\right)$mRNA was purchased from Ambion (Cat. \#7849). Mouse spleen total RNA was purchased from Ambion (Cat. \#7920). A20 and R1.1 cell lines were purchased from the American Type Culture Collection (ATCC; A20, Cat. \#TIB-208, R1.1, Cat. \#TIB-42, R1.1) and were grown according to supplier's recommendations. A20 cells were grown in RPMI 1640 medium with $2 \mathrm{mM}$ L-glutamine, and supplemented with $1.5 \mathrm{~g} / \mathrm{L} \mathrm{NaHCO}_{3}, 1.0 \mathrm{mM}$ Na pyruvate, 10 $\mathrm{mM}$ HEPES, and 10\% fetal bovine serum (Hyclone). R1.1 cells were grown in DMEM high-glucose medium with glutamate supplemented with $1.5 \mathrm{~g} / \mathrm{L} \mathrm{NaHCO}_{3}$ and $10 \%$ horse serum. Total RNA was harvested from $\sim 10^{8}$ cells using the RNeasy Midi kit (QIAGEN) according to the manufacturer's instructions.

\section{Labeling}

Although our platform is amenable to a number of standard sample labeling techniques, our preferred approach is based on the modified Eberwine protocol (Eberwine et al. 1992), by which messenger RNA is converted to cDNA, followed by an amplification/labeling step mediated by T7 DNA polymerase. The linear amplification step reduces the amount of starting material needed. We adapted the protocol to a microtiter plate format in order to match the array matrix format, which permits 96 array hybridizations to be performed in parallel. Labeling and amplification of the total RNA samples were performed according to the MessageAmp aRNA kit (Ambion Cat. \#1750) with the following modifications. Because the hybridization requirements are so modest ( $1 \mu \mathrm{g}$ labeled cRNA), the standard reaction was cut down to 1/4 size and total RNA inputs were generally limited to $100 \mathrm{ng}$. The use of smaller reactions allowed us to perform 80 reactions per kit as opposed to the standard 20 reactions. This necessitated the use of additional cleanup columns for both the RT and IVT steps. QIAquick PCR Purification and RNeasy 96 well kits (QIAGEN) were used according to the manufacturer's instructions for RT and IVT cleanup, respectively. Additionally, all components of the first-strand cDNA synthesis were combined in a single step,

\footnotetext{
${ }^{3}$ Standard, semicustom and fully custom bead sets are provided commercially by Illumina. Semicustom bead sets are made by supplementing standard sets with sequences of the customer's choosing. Fully custom bead sets can be made from any desired set of sequences.
}

\section{Genome Research}

www.genome.org 
Random arrays for gene expression profiling

A

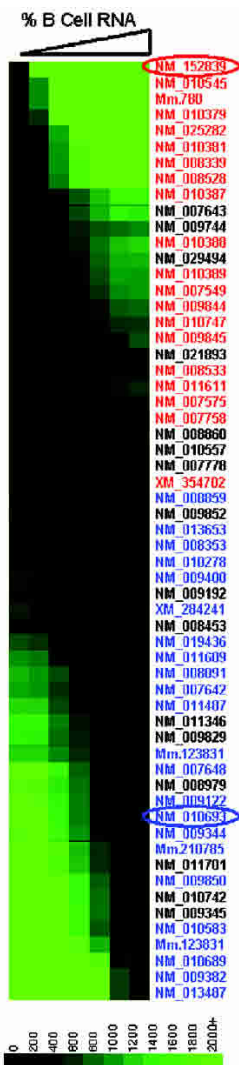

B

$\lg \mathbf{J}$ (Immunoglobulin joining chain)

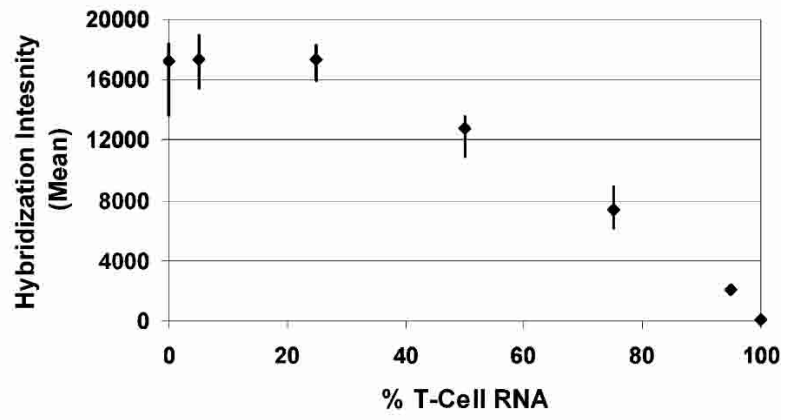

Ick (lymphocyte protein tyrosine kinase)

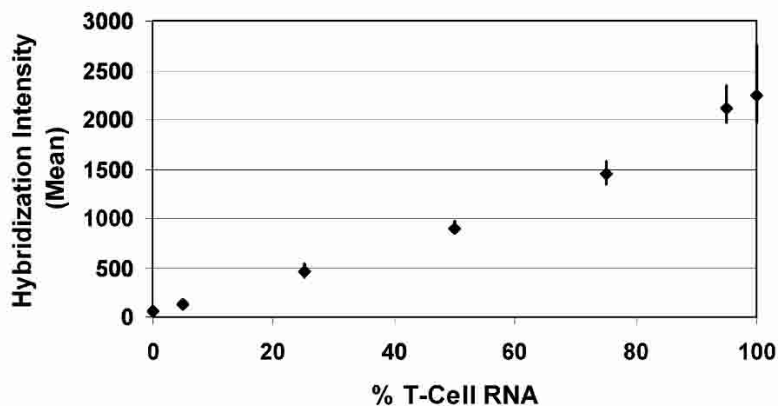

Figure 8. B Cell/T Cell experimental results. Seven RNA samples were prepared containing mixtures of B- and T-cell lymphoma cell-line mRNA. The samples contained $0 \%, 5 \%, 25 \%, 50 \%$, $95 \%$, and $100 \%$ B-cell RNA, with the balance in all cases being T-cell RNA. These samples were labeled by our standard protocol and hybridized to six separate arrays each. $(A)$ The hybridization intensities of the 59 most tissue-specific genes are plotted. Each vertical stripe represents a sample and each horizontal row a gene. Boxes represent the mean hybridization intensities measured in six replicate array hybridizations. The intensity scale is shown in the legend at the bottom. Genes are labeled according to their RefSeq or UniGene ID numbers. The colors of these labels indicate prior evidence in the literature of B- or T-cell-specific expression (red refers to B-cell-specific expression; blue refers to T-cell-specific; see Table 2 for references). (B) The dose responses of two representative genes identified in this experiment are plotted. Points represent the mean intensities for each concentration. Error bars represent $90 \%$ two-sided confidence intervals calculated from six replicate hybridizations.

because a separate annealing of the $\mathrm{T} 7$ oligo(dT) primer was found to be unnecessary (data not shown). During the IVT reaction, a 1:1 ratio of labeled bio-16-UTP (Roche Cat. \#1388908) to unlabeled UTP was used with a final combined concentration of $7.5 \mathrm{mM}$.

\section{Preparation of labeled spikes}

Nine bacterial and viral genes were used to prepare RNA controls as follows: bla (pBluescriptSK+; Stratagene) cat (pCAT3-control; Promega), cre (Escherichia coli DH10B-Zip; Life Technologies), e1a (Homo sapiens HEK-293; ATCC), gfp (pEGFP; Clontech), gst (pGEX-5x-3; Amersham-Pharmacia), gus (E. coli GM48), lux (E. coli GM48), and neo (pGT-N28; New England Biolabs). The genes were cloned into the PCRII cloning vector using the TA Cloning kit (Invitrogen, Cat. \#K205001-TA). Full-length sense transcripts were generated using the MEGA-script T3 kit from Ambion (Cat. \#1338). Labeled antisense targets were then generated using the MessageAmp aRNA kit and were spiked into labeled Human HepG 2 cRNA at the 12 concentrations shown in Figure 2.
Hybridization / washing/signal detection

All steps of hybridization, washing, blocking, and signal generation were performed by sequential transfer of a Sentrix array matrix from one 384-well plate (ThermoLab Systems; Cat. \#95040000) to the next with the wells of each step containing $40 \mu \mathrm{L}$ of the appropriate solution. All incubations were carried out without agitation and, with the exception of the hybridization, at room temperature. Amplified, biotinlabeled human or mouse RNA samples were prepared in a solution of Hyb E1 buffer (Illumina, Part \#11166381) and 25\% (v/v) formamide at a final concentration of $25 \mathrm{ng} /$ $\mu \mathrm{L}$. An array matrix was then mated to the hybridization plate using a sealed alignment fixture. Hybridization proceeded at $55^{\circ} \mathrm{C}$, for 16 to $20 \mathrm{~h}$. After hybridization, the array matrix was washed by a 5 -min incubation in Illumina Wash E1 buffer, followed by a 10-min wash in fresh Wash E1 buffer (Illumina, Part \#11165898). Arrays were then blocked for $5 \mathrm{~min}$ in $1 \%(\mathrm{w} / \mathrm{v})$ casein-PBS, Hammerstein grade (Pierce, Cat. \#37528). Array signal was developed by a 10 -min incubation in a $1-\mu \mathrm{g} / \mathrm{mL}$ solution of Streptavidin-Cy3 (Amersham; Cat. \#PA43001) in 1\% casein-PBS blocking solution. The array matrix was washed a final time for $5 \mathrm{~min}$ in Wash E1 buffer. Each array was then dried with an air gun.

\section{Imaging and signal extraction}

Arrays were scanned on the BeadArray Reader, a confocal-type imaging system with $\sim 0.8 \mu \mathrm{m}$ resolution and 532 and 635 $\mathrm{nm}$ laser illumination (Barker et al. 2003). Scans were performed in the 532-nm channel. The total scan time per array matrix (i.e., 96 arrays) was $1.5 \mathrm{~h}$, roughly $1 \mathrm{~min}$ per array. Image analysis and data extraction software were as described previously (Fan et al. 2003). Briefly, each sequence type is represented by an average of 30 beads on the array. Bead signals were computed with weighted averages of pixel intensities, and local background was subtracted. Array images are registered by a previously described algorithm (Galinsky 2003). This algorithm supplies the position of a bead center that serves as a center for a virtual pixel. To compute bead signal, we use four real pixels covering the virtual one and combine their signals in the following way: $S=A 1 S 1+A 2 S 2+A 3 S 3+A 4 S 4$, where $S$ is bead signal, $A i$ is area of overlap between ith pixel and the virtual pixel, and $\mathrm{Si}$ is $3 \times 3$ average taken around ith pixel after sharpening with following Laplacian:

$$
I_{x, y}^{\text {sharp }}=I_{x, y}+0.5\left(4 I_{x, y}-I_{x, y+1}-I_{x, y-1}-I_{x+1, y}-I_{x-1, y}\right) .
$$

Here $x, y$ are pixel coordinates and $I x, y$ are pixel intensities. The choice of coefficient in front of Laplacian was made after optimization of data obtained with calibrated set of Spherotech 3-micron rainbow beads (Cat. \#RCP-30-5, Spherotech, Inc.). Finally, we subtract local background as average of five dimmest pixels in the $17 \times 17$ box centered in the pixel having maximum overlap with the virtual pixel. Sequence-type signal was calculated by 
Kuhn et al.

Table 2. Array-based determination of tissue-specific gene expression

\begin{tabular}{|c|c|c|c|c|c|}
\hline $\begin{array}{l}\text { Gene RefSeq or } \\
\text { UniGene ID }\end{array}$ & $\begin{array}{c}\text { HUGO } \\
\text { Gene Symbol }\end{array}$ & Gene Name & $\begin{array}{l}\text { T/B Cell Ratio } \\
\text { by array }\end{array}$ & $\begin{array}{l}\text { B or T-specific } \\
\text { by literature }\end{array}$ & Ref $^{c}$ \\
\hline NM_010545 & li & la-associated invariant chain & 0.003 & B & 1 \\
\hline NM_152839 & Igj & immunoglobulin joining chain & 0.004 & B & 1 \\
\hline NM_008339 & Cd79b & CD79B antigen & 0.006 & B & 1 \\
\hline $\mathrm{Mm} .780$ & Igl-V1 & immunoglobulin $\lambda$ chain, variable 1 & 0.007 & B & 1 \\
\hline NM_010381 & $\mathrm{H} 2-\mathrm{Ea}$ & histocompatibility 2 , class II antigen $\mathrm{E} \alpha$ & 0.008 & B & 1 \\
\hline NM_008860 & Prkcz & protein kinase $C, \zeta$ & 0.010 & - & \\
\hline NM_010379 & H2-Ab1 & histocompatibility 2 , class II antigen $A, \beta 1$ & 0.010 & B & 1 \\
\hline NM_008528 & Blnk & B-cell linker & 0.010 & B & 1 \\
\hline NM_025282 & Mef2c & myocyte enhancer factor $2 \mathrm{C}$ & 0.011 & B & 2 \\
\hline NM_010389 & $\mathrm{H} 2-\mathrm{Ob}$ & histocompatibility $2, O$ region $\beta$ locus & 0.011 & B & 1 \\
\hline NM_009844 & Cd19 & CD19 antigen & 0.011 & B & 1 \\
\hline NM_007643 & $\mathrm{Cd} 36$ & CD36 antigen & 0.015 & - & \\
\hline NM_010387 & H2-DMb1 & histocompatibility 2, class II, locus Mb1 & 0.024 & B & 1 \\
\hline NM_010388 & H2-DMb2 & histocompatibility 2, class II, locus Mb2 & 0.037 & B & 1 \\
\hline NM_007549 & Blk & B lymphoid kinase & 0.038 & B & 1 \\
\hline NM_029494 & Rsb30 & RAB30, member RAS oncogene family & 0.041 & - & \\
\hline NM_009845 & $\mathrm{Cd} 22$ & CD22 antigen & 0.051 & B & 1 \\
\hline NM_011611 & Tnfrsf5 & tumor necrosis factor receptor superfamily, member 5 & 0.062 & B & 1 \\
\hline NM_010747 & Lyn & Yamaguchi sarcoma viral (v-yes-1) oncogene homolog & 0.065 & B & 3 \\
\hline NM_007575 & $\mathrm{C} 2 \mathrm{ta}$ & class II transactivator & 0.088 & B & 1 \\
\hline NM_008533 & Ly78 & lymphocyte antigen 78 & 0.088 & B & 1 \\
\hline NM_009744 & $\mathrm{Bcl} 6$ & B-cell leukemia/lymphoma 6 & 0.106 & - & \\
\hline NM_007758 & $\mathrm{Cr} 2$ & complement receptor 2 & 0.107 & B & 4 \\
\hline XM_354702 & none & similar to immunoglobulin $\varepsilon$ & 0.110 & B & 1 \\
\hline NM_021893 & Pdcd1 lg1 & programmed cell death 1 ligand 1 & 0.126 & - & \\
\hline NM 010557 & $\| 4 \mathrm{ra}$ & interleukin 4 receptor, $\alpha$ & 0.142 & - & \\
\hline NM_007778 & Csf1 & colony stimulating factor 1 (macrophage) & 0.226 & - & \\
\hline NM_009852 & $\mathrm{Cd} 6$ & CD6 antigen & 4.70 & - & \\
\hline NM_013653 & $\mathrm{Ccl} 5$ & chemokine (C-C motif) ligand 5 & 7.72 & $\mathrm{~T}$ & 5 \\
\hline XM_284241 & Tnfrsf7 & tumor necrosis factor receptor superfamily, member 7 & 10.35 & $\mathrm{~T}$ & 1 \\
\hline NM 008453 & Klf3 & Kruppel-like factor 3 (basic) & 10.70 & - & \\
\hline NM_009400 & Tnfrsf18 & tumor necrosis factor receptor superfamily, member 18 & 14.82 & $\mathrm{~T}$ & 6 \\
\hline NM_009192 & Sla & src-like adaptor & 16.72 & - & \\
\hline NM_008353 & ॥l12rb1 & interleukin 12 receptor, $\beta 1$ & 20.91 & $\mathrm{~T}$ & 1 \\
\hline NM_019436 & Sit & SHP2 interacting transmembrane adaptor & 26.66 & $\mathrm{~T}$ & 1 \\
\hline NM_008859 & Prkcq & protein kinase $C, \theta$ & 27.05 & $\mathrm{~T}$ & 7 \\
\hline NM_010742 & Ly6d & lymphocyte antigen 6 complex, locus D & 31.17 & - & \\
\hline Mm. 123831 & Tcrb-V8.2 & T-cell receptor $\beta$, variable 8.2 & 35.75 & $\mathrm{~T}$ & 1 \\
\hline NM 008091 & Gata3 & GATA binding protein 3 & 40.45 & $\mathrm{~T}$ & 1 \\
\hline NM_010693 & Lck & lymphocyte protein tyrosine kinase & 40.51 & $\mathrm{~T}$ & 1 \\
\hline NM_010278 & Gfi1 & growth factor independent 1 & 42.67 & $\mathrm{~T}$ & 8 \\
\hline NM_011487 & Stat4 & signal transducer and activator of transcription 4 & 49.24 & $\mathrm{~T}$ & 1 \\
\hline NM_011701 & Vim & Vimentin & 52.19 & - & \\
\hline NM_009122 & Satb1 & special AT-rich sequence binding protein 1 & 56.90 & $\mathrm{~T}$ & 9 \\
\hline NM_009850 & $C d 3 g$ & CD3 antigen, $\gamma$ polypeptide & 59.09 & $\mathrm{~T}$ & 1 \\
\hline NM_009344 & Phlda1 & pleckstrin homology-like domain, family A, member 1 & 63.57 & $\mathrm{~T}$ & 10 \\
\hline NM 007642 & $\mathrm{Cd} 28$ & CD28 antigen & 67.64 & $\mathrm{~T}$ & 1 \\
\hline NM_011346 & Sell & selectin, lymphocyte & 72.79 & - & \\
\hline NM_007648 & Cd3e & $\mathrm{CD} 3$ antigen, $\varepsilon$ polypeptide & 93.03 & $\mathrm{~T}$ & 1 \\
\hline NM_008979 & Ptpn8 & protein tyrosine phosphatase, non-receptor type 8 & 113.63 & - & \\
\hline NM_009345 & Dntt & deoxynucleotidyltransferase, terminal & 118.86 & - & \\
\hline NM_010583 & Itk & IL2-inducible T-cell kinase & 120.75 & $\mathrm{~T}$ & 11 \\
\hline NM_011609 & Tnfrsf1a & tumor necrosis factor receptor superfamily, member $1 \mathrm{a}$ & 132.72 & - & \\
\hline Mm. 123831 & Tcrb-V13 & T-cell receptor $\beta$, variable & 167.67 & $\mathrm{~T}$ & 1 \\
\hline NM_010689 & Lat & linker for activation of $\mathrm{T}$ cells & 168.24 & $\mathrm{~T}$ & 12 \\
\hline NM_009382 & Thy1 & thymus cell antigen $1, \theta$ & 171.82 & $\mathrm{~T}$ & 1 \\
\hline NM_009829 & Cond2 & cyclin D2 & 242.81 & - & \\
\hline Mm.210785 & & T cell receptor $\gamma$ chain & 424.47 & $\mathrm{~T}$ & 1 \\
\hline NM_013487 & $C d 3 d$ & CD3 antigen, $\delta$ polypeptide & 1532.15 & $\mathrm{~T}$ & 1 \\
\hline
\end{tabular}

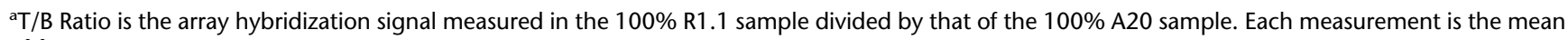
of four separate arrays.

${ }^{\mathrm{b}}$ According to literature. Blanks indicate that we could not find literature-based evidence for T- or B-cell-specific expression. For 14 of the $17 \mathrm{genes}$ for which there was no literature support for the expression pattern, the same samples have been examined by a reverse transcription-based RNA quantitation assay (Fan et al. 2004); in all cases examined, the results from this orthogonal assay agreed with the results determined in this array experiment (J. Yeakley, J.-B. Fan, and E. Chudin, unpubl.).

'References: Abbas et al. (2003); Hermanson et al. (1988); Yamanashi et al. (1991); Fingeroth (1990); Schall et al. (1988); Nocentini et al. (1997); Isakov and Altman (2002); Scheijen et al. (1997); Dickinson et al. (1992); Park et al. (1996); Siliciano et al. (1992); Zhang et al. (1998). 
Random arrays for gene expression profiling

averaging corresponding bead signals with outliers removed (using median absolute deviation).

\section{Data analysis}

We developed a suite of algorithms for analysis of gene expression data from microarrays (E. Chudin and I. Mikoulitch, pers. comm.). These have been incorporated into AnEx, a commercial software package for gene-expression data analysis. Array data were normalized using quantiles to fit a cubic spline. The approach is similar to a previously reported method (Workman et al. 2002). Alternatively, a robust least-squares fit (iteratively reweighted least squares using Tukey's biweight functions) of intensities of a rank invariant set of probes (relative rank change of $<0.05$ ) was used. Detection $p$-values were computed using a dynamically constructed normal model based on intensities of 20 negative controls. To determine minimal resolvable fold change, we used piecewise linear approximation of intensity versus concentration. Concentration levels were considered resolvable if corresponding one-sided 95th percent confidence intervals, as computed from t-distribution did not overlap. Piecewise linear interpolation was used for both intensities and standard deviations.

\section{Array design}

Probes were designed by a custom-built pipeline that will be described in detail elsewhere (P. Rigault, in prep.). Each gene sequence for which probes were to be synthesized was subjected to a filtering process that masked regions unsuitable for probe design, based on complexity and cross-homology thresholds, as determined by DUST (D. Lipman, National Center for Biotechnology Information, pers. comm.) and BLAST (Altschul et al. 1990) algorithms, respectively. All possible 50-mer probes were identified within unmasked regions, and these were ranked by a formula that takes into account distance from the 3' end of the transcript, melting temperature, and self-complementarity. The two highest scoring probes were then linked to 23-nt identifier sequences by use of a sequence-matching program that minimizes the probability of interactions between the probe and identifier sequence and prevents the creation of junction sequences with cross-homology to the genome in question.

Our use of two probes per gene was based on the results of pilot experiments, in which five informatically chosen probes were synthesized for each of 10 in vitro-synthesized genes. Dose response was determined for each synthetic gene using all five probes or four, three, two, or one arbitrarily selected probes. We found that we could reach our targeted performance metrics (Table 1) with two or more probes per gene, but not one (data not shown). The results of recent functional screening suggests that one probe per gene is sufficient if the probes are selected with a functional screen (T. McDaniel, B. Kermani, S. Baker, S. Oeser, and S. Kruglyak, unpubl.).

\section{Quantitative PCR}

Assays-on-Demand quantitative gene expression primers and TaqMan universal PCR master mix (Cat. \#4304437) were purchased from Applied Biosystems. All PCR reactions were performed following the manufacturer's instructions.

\section{Acknowledgments}

We thank Steven Barnard, Chanfeng Zhao, Paul Kitabjian, Michael Graige, and Semyon Kruglyak for devising methods to prepare beads with gene-specific probes and for providing bead pools used in these experiments. We also thank Chan Tsan for technical assistance, Lixin Zhou for help with analysis of the B and $T$ cell experiments, and the array manufacturing group at Illumina for providing gene-specific probe arrays.

\section{References}

Abbas, A.K., Lichtman, A.H., and Pober, J.S. 2003. Cellular and molecular immunology. W.B. Saunders, Philadelphia, PA.

Altschul, S.F., Gish, W., Miller, W., Myers, E.W., and Lipman, D.J. 1990. Basic local alignment search tool. J. Mol. Biol. 215: 403-410.

Barker, D.L., Therault, G., Che, D., Dickinson, T., Shen, R., and Kain, R. 2003. Self-assembled random arrays: High-performance imaging and genomics applications on a high-density microarray platform. Proc. SPIE 4966: 1-11.

Blanchard, A. 1998. Synthetic DNA arrays. Plenum Press, New York.

Brody, J.P., Williams, B.A., Wold, B.J., and Quake, S.R. 2002. Significance and statistical errors in the analysis of DNA microarray data. Proc. Natl. Acad. Sci. 99: 12975-12978.

Dickinson, L.A., Joh, T., Kohwi, Y., and Kohwi-Shigematsu, T. 1992. A tissue-specific MAR/SAR DNA-binding protein with unusual binding site recognition. Cell 70: 631-645.

Eberwine, J., Yeh, H., Miyashiro, K., Cao, Y., Nair, S., Finnell, R., Zettel, M., and Coleman, P. 1992. Analysis of gene expression in single live neurons. Proc. Natl. Acad. Sci. 89: 3010-3014.

Epstein, J.R., Lee, M., and Walt, D.R. 2002. High-density fiber-optic genosensor microsphere array capable of zeptomole detection limits. Anal. Chem. 74: 1836-1840.

Fan, J.-B., Oliphant, A., Shen, R., Kermani, B.G., Garcia, F., Gunderson, K.L., Hansen, M., Steemers, F., Butler, S.L., Deloukas, P., et al. 2003. Highly parallel SNP genotyping. Cold Spring Harbor Symp. Biol. 68: $69-78$.

Fan, J.B., Yeakley, J.M., Bibikova, M., Chudin, E., Wickham, E., Chen, J., Doucet, D., Rigault, P., Zhang, B., Shen, R., et al. 2004. A versatile assay for high-throughput gene expression profiling on universal array matrices. Genome Res. 14: 878-885.

Fingeroth, J.D. 1990. Comparative structure and evolution of murine CR2. The homolog of the human C3d/EBV receptor (CD21). J. Immunol. 144: 3458-3467.

Fodor, S.P.A., Read, J.L., Pirrung, M.C., Stryer, L., Lu, A.T., and Solas, D. 1991. Light-directed, spatially addressable parallel chemical synthesis. Science 251: 767-773.

Galinsky, V.L. 2003. Automatic registration of microarray images. II. Hexagonal grid. Bioinformatics 19: 1832-1836.

Golub, T.R., Slonim, D.K., Tamayo, P., Huard, C., Gaasenbeek, M., Mesirov, J.P., Coller, H., Loh, M.L., Downing, J.R., Caligiuri, M.A., et al. 1999. Molecular classification of cancer: Class discovery and class prediction by gene expression monitoring. Science 286: 531-537.

Gunderson, K., Kruglyak, S., Graige, M.S., Garcia, F., Kermani, B.G., Zhao, C., Che, D., Milewski, M., Yang, R., Siegmund, C., et al. 2004. Decoding randomly ordered arrays. Genome Res. 14: 870-877.

Heid, C.A., Stevens, J., Livak, K.J., and Williams, P.M. 1996. Real time quantitative PCR. Genome Res. 6: 986-994.

Hermanson, G.G., Eisenberg, D., Kincade, P.W., and Wall, R. 1988. B29: A member of the immunoglobulin gene superfamily exclusively expressed on $\beta$-lineage cells. Proc. Natl. Acad. Sci. 85: 6890-6894.

Hughes, T.R., Marton, M.J., Jones, A.R., Roberts, C.J., Stoughton, R., Armour, C.D., Bennett, H.A., Coffey, E., Dai, H., He, Y.D., et al. 2000 . Functional discovery via a compendium of expression profiles. Cell 102: 109-126.

Hughes, T.R., Mao, M., Jones, A.R., Burchard, J., Marton, M.J., Shannon, K.W., Lefkowitz, S.M., Ziman, M., Schelter, J.M., Meyer, M.R., et al. 2001. Expression profiling using microarrays fabricated by an ink-jet oligonucleotide synthesizer. Nat. Biotechnol. 19: 342-347.

Ideker, T., Thorsson, V., Ranish, J.A., Christmas, R., Buhler, J., Eng, J.K., Bumgarner, R., Goodlett, D.R., Aebersold, R., and Hood, L. 2001. Integrated genomic and proteomic analyses of a systematically perturbed metabolic network. Science 292: 929-934.

The International HapMap Consortium. 2003. The International HapMap Project. Nature 426: 789-796.

Isakov, N. and Altman, A. 2002. Protein kinase C(theta) in T cell activation. Annu. Rev. Immunol. 20: 761-794.

Lockhart, D.J. and Winzeler, E.A. 2000. Genomics, gene expression and DNA arrays. Nature 405: 827-836.

Lockhart, D.J., Dong, H., Byrne, M.C., Follettie, M.T., Gallo, M.V., Chee, M.S., Mittmann, M., Wang, C., Kobayashi, M., Horton, H., et al. 1996. Expression monitoring by hybridization to high-density oligonucleotide arrays. Nat. Biotechnol. 14: 1675-1680.

Marton, M.J., DeRisi, J.L., Bennett, H.A., Iyer, V.R., Meyer, M.R., Roberts, C.J., Stoughton, R., Burchard, J., Slade, D., Dai, H., et al. 1998. Drug 


\section{Kuhn et al.}

target validation and identification of secondary drug target effects using DNA microarrays. Nat. Med. 4: 1293-1301.

Michael, K.L., Taylor, L.C., Schultz, S.L., and Walt, D.R. 1998. Randomly ordered addressable high-density optical sensor arrays. Anal. Chem. 70: $1242-1248$.

Nocentini, G., Giunchi, L., Ronchetti, S., Krausz, L.T., Bartoli, A., Moraca, R., Migliorati, G., and Riccardi, C. 1997. A new member of the tumor necrosis factor/nerve growth factor receptor family inhibits T cell receptor-induced apoptosis. Proc. Natl. Acad. Sci. 94: $6216-6221$.

Park, C.G., Lee, S.Y., Kandala, G., and Choi, Y. 1996. A novel gene product that couples TCR signaling to Fas(CD95) expression in activation-induced cell death. Immunity 4: 583-591.

Schall, T.J., Jongstra, J., Dyer, B.J., Jorgensen, J., Clayberger, C., Davis, M.M., and Krensky, A.M. 1988. A human T cell-specific molecule is a member of a new gene family. J. Immunol. 141: 1018-1025.

Scheijen, B., Jonkers, J., Acton, D., and Berns, A. 1997. Characterization of pal-1, a common proviral insertion site in murine leukemia virus-induced lymphomas of c-myc and Pim-1 transgenic mice. $J$. Virol. 71: 9-16.

Schena, M., Shalon, D., Davis, R.W., and Brown, P.O. 1995. Quantitative monitoring of gene expression patterns with a complementary DNA microarray. Science 270: 467-470.

Siliciano, J.D., Morrow, T.A., and Desiderio, S.V. 1992. itk, a T-cell-specific tyrosine kinase gene inducible by interleukin 2. Proc. Natl. Acad. Sci. 89: 11194-11198.

van't Veer, L.J., Dai, H., van de Vijver, M.J., He, Y.D., Hart, A.A., Mao, M., Peterse, H.L., van der Kooy, K., Marton, M.J., Witteveen, A.T., et al. 2002. Gene expression profiling predicts clinical outcome of breast cancer. Nature 415: 530-536.

Workman, C., Jensen, L.J., Jarmer, H., Berka, R., Gautier, L., Nielser,

H.B., Saxild, H.H., Nielsen, C., Brunak, S., and Knudsen, S. 2002. A new non-linear normalization method for reducing variability in DNA microarray experiments. Genome Biol. 3: research0048.

Yamanashi, Y., Kakiuchi, T., Mizuguchi, J., Yamamoto, T., and Toyoshima, K. 1991. Association of B cell antigen receptor with protein tyrosine kinase Lyn. Science 251: 192-194.

Yeakley, J.M., Fan, J.B., Doucet, D., Luo, L., Wickham, E., Ye, Z., Chee, M.S., and Fu, X.D. 2002. Profiling alternative splicing on fiber-optic arrays. Nat. Biotechnol. 20: 353-358.

Yvert, G., Brem, R.B., Whittle, J., Akey, J.M., Foss, E., Smith, E.N., Mackelprang, R., and Kruglyak, L. 2003. Trans-acting regulatory variation in Saccharomyces cerevisiae and the role of transcription factors. Nat. Genet. 35: 57-64.

Zhang, W., Sloan-Lancaster, J., Kitchen, J., Trible, R.P., and Samelson, L.E. 1998. LAT: The ZAP-70 tyrosine kinase substrate that links T cell receptor to cellular activation. Cell 92: 83-92.

\section{Web site references}

www.hapmap.org; International HapMap Project.

www.illumina.com; Illumina, Inc.

www.mged.org; Microarray Gene Expression Data Society.

Received April 30, 2004; accepted in revised form August 16, 2004. 


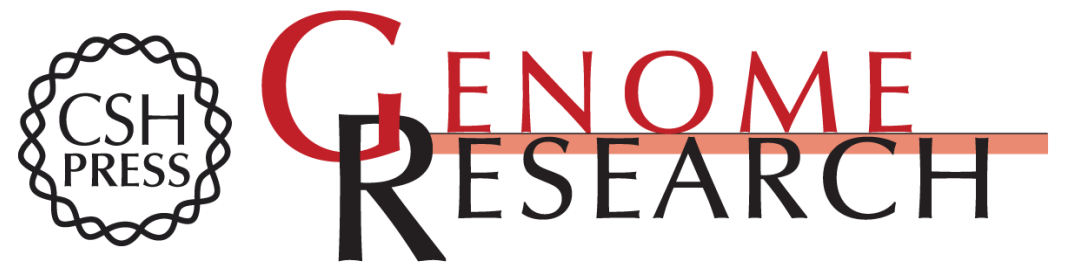

\section{A novel, high-performance random array platform for quantitative gene expression profiling}

Kenneth Kuhn, Shawn C. Baker, Eugene Chudin, et al.

Genome Res. 2004 14: 2347-2356

Access the most recent version at doi:10.1101/gr.2739104

References This article cites 34 articles, 16 of which can be accessed free at:

http://genome.cshlp.org/content/14/11/2347.full.html\#ref-list-1

\section{License}

Email Alerting Receive free email alerts when new articles cite this article - sign up in the box at the Service top right corner of the article or click here.

\section{Affordable, Accurate Sequencing.}

To subscribe to Genome Research go to: https://genome.cshlp.org/subscriptions 